\title{
BEST CONSTANTS IN NORM INEQUALITIES FOR THE DIFFERENCE OPERATOR
}

\author{
HANS G. KAPER AND BETH E. SPELLMAN
}

\begin{abstract}
Let $\xi=\left(\xi_{m}\right)_{m \in \mathbf{Z}}$ be an arbitrary element of the sequence space $l^{\infty}(\mathbf{Z})$, and let $\Delta$ be the difference operator on $l^{\infty}(\mathbf{Z}): \Delta \xi=\left(\xi_{m+1}-\xi_{m}\right)_{m \in \mathbf{Z}}$. The object of this investigation is the best possible value

$$
C(n, k)=\sup \left\{Q_{n, k}(\xi): \xi \in l^{\infty}(\mathbf{Z}), \Delta^{n} \xi \neq 0\right\}
$$

of the quotient

$$
Q_{n, k}(\xi)=\frac{\left\|\Delta^{k} \xi\right\|}{\|\xi\|^{(n-k) / n}\left\|\Delta^{n} \xi\right\|^{k / n}},
$$

where $n=2,3, \ldots ; k=1, \ldots, n-1$. It is shown that $C(n, k)$ is at least equal to the corresponding constant $K(n, k)$, determined by Kolmogorov [Moscov. Gos. Univ. Uchen. Zap. Mat. 30 (1939), 3-13; Amer. Math. Soc. Transl. (1) 2 (1962), 233-243] for the differential operator $D$ on $L^{\infty}(\mathbf{R})$, and exactly equal to $K(n, k)$ if $k=n-1$. Lower bounds for $C(n, k)$ are derived that show that $C(n, k)$ is generally greater than $K(n, k)$. The values of $C(n, k), k=1, \ldots, n-1$, are computed for $n=2, \ldots, 5$.
\end{abstract}

1. Introduction. In 1914, Hadamard [1] showed that if the twice differentiable real-valued function $f$ on $(-\infty, \infty)$ is bounded along with its second derivative, then

$$
\mu_{1} \leqslant \sqrt{2} \mu_{0}^{1 / 2} \mu_{2}^{1 / 2}
$$

where $\mu_{j}=\sup \left\{\left|f^{(j)}(x)\right|:-\infty<x<\infty\right\}, j=0,1,2$. This result was the analog of a similar inequality derived earlier by Landau [2] for functions $f$ on $(0, \infty)$, viz.,

$$
\mu_{1} \leqslant 2 \mu_{0}^{1 / 2} \mu_{2}^{1 / 2}
$$

where $\mu_{j}=\sup \left\{\left|f^{(j)}(x)\right|: 0 \leqslant x<\infty\right\}, j=0,1,2$.

The classical inequality (1.1) was generalized to higher-order derivatives first by Šilov [3] for derivatives up to order five and, subsequently, by Kolmogorov [4, 5] for derivatives of arbitrary order. In [5], Kolmogorov showed that if the $n$ times continuously differentiable real-valued function $f$ on $(-\infty, \infty)$ is bounded along

Received by the editors November 8, 1985. Presented at the 92nd Annual Meeting of the American Mathematical Society, New Orleans, January, 1986 (825-39-564).

1980 Mathematics Subject Classification (1985 Revision). Primary 39A70, 47B39; Secondary 39A12, $41 \mathrm{~A} 44$.

Key words and phrases. Best constants, norm inequalities, difference operator, powers of operators, sequence spaces, Kolmogorov constants, spline functions.

This work was supported by the Applied Mathematical Sciences subprogram of the Office of Energy Research, U. S. Department of Energy, under contract W-31-109-Eng-38.

The second author was a participant in the Summer Student Research Program.

C1987 American Mathematical Society $0002-9947 / 87 \$ 1.00+\$ .25$ per page 
with its $n$th derivative $(n=2,3, \ldots)$, then

$$
\mu_{k} \leqslant K(n, k) \mu_{0}^{(n-k) / n} \mu_{n}^{k / n}, \quad k=1, \ldots, n-1,
$$

where $\mu_{j}=\sup \left\{\left|f^{(j)}(x)\right|:-\infty<x<\infty\right\}, j=0, \ldots, n$. The constants $K(n, k)$ are best possible; they are given by

$$
K(n, k)=\frac{M_{n-k}}{M_{n}^{(n-k) / n}},
$$

where

$$
M_{j}=\frac{4}{\pi} \sum_{p=0}^{\infty} \frac{(-1)^{(j+1) p}}{(2 p+1)^{j+1}} .
$$

Extremal functions, for which equality is attained in (1.3), are of the form $f_{n k}(x)=$ $c \varphi_{n}(\rho x)$, where $c$ and $\rho$ are constants and

$$
\varphi_{n}(x)=\frac{4 n !}{\pi^{n+1}} \sum_{p=0}^{\infty} \frac{\sin ((2 p+1) \pi x-n \pi / 2)}{(2 p+1)^{n+1}} .
$$

Later, it was observed by Ljubič $[6,7]$ that inequality $(1.3)$ is a particular instance of a more general class of inequalities involving the norms of powers of linear operators. Thus, if $D$ is the differential operator with maximal domain in $L^{\infty}(\mathbf{R})$, the best possible constant $K(n, k)$ in (1.3) is given by

$$
K(n, k)=\sup \left\{Q_{n, k}(f): f \in \operatorname{dom} D^{n} \subset L^{\infty}(\mathbf{R}), D^{n} f \neq 0\right\},
$$

where

$$
Q_{n, k}(f)=\frac{\left\|D^{k} f\right\|}{\|f\|^{(n-k) / n}\left\|D^{n} f\right\|^{k / n}} .
$$

The same observation motivated Gindler and Goldstein [8, 9] in their investigations of inequalities involving the norms of powers of dissipative operators on Banach spaces.

The discrete analog of $D$ is the difference operator $\Delta$ on the sequence space $l^{\infty}(\mathbf{Z})$. If $\xi=(\xi)_{m \in \mathbf{Z}}$ denotes the general element of $l^{\infty}(\mathbf{Z})$, then $\Delta \xi$ is the vector with components

$$
(\Delta \xi)_{m}=\xi_{m+1}-\xi_{m}, \quad m \in \mathbf{Z} .
$$

$\Delta$ is a bounded operator on $l^{\infty}(\mathbf{Z})$, whose norm is 2 . Successive powers of $\Delta$ are defined in the usual way,

$$
\Delta^{k} \xi=\Delta\left(\Delta^{k-1} \xi\right), \quad k=2,3, \ldots
$$

In the present article, we are interested in the analogs of Kolmogorov's best constants $K(n, k)$ for the difference operator $\Delta$. Denoting them by $C(n, k)$, we have

$$
C(n, k)=\sup \left\{Q_{n, k}(\xi): \xi \in l^{\infty}(\mathbf{Z}), \Delta^{n} \xi \neq 0\right\},
$$

where

$$
Q_{n, k}(\xi)=\frac{\left\|\Delta^{k} \xi\right\|}{\|\xi\|^{(n-k) / n}\left\|\Delta^{n} \xi\right\|^{k / n}}
$$


These constants have been investigated previously by Ditzian [10] and, more recently, by Ditzian and Newman [11].

In $\$ 2$ we explore some corollaries of Kolmogorov's results for the best constants $K(n, k)$. In $\S 3$ we show that the discrete constants $C(n, k)$ are at least equal to the corresponding $K(n, k)$, and exactly equal if $k=n-1$. In $\S 4$ we construct lower bounds for the discrete constants $C(n, k)$ and show that $C(n, k)$ is generally greater than the corresponding $K(n, k)$. In $\$ 5$ we derive several inequalities for the quotient $Q_{n, k}(\xi)$. In $\S 6$ we compute the values of $C(n, k), k=1, \ldots, n-1$, for $n=2, \ldots, 5$.

2. Kolmogorov's results revisited. The extremals $\varphi_{n}$ given in (1.6) are examples of (unscaled and shifted) Euler splines (cf. [12, Lecture 4 or 13, Chapter VI]).

Let the polynomial functions $E_{n}, n=1,2, \ldots$, be defined on $[0,1]$ recursively by the relation

$$
E_{n}^{\prime}(x)=n E_{n-1}(x), \quad E_{n}(0)+E_{n}(1)=0, \quad n=1,2, \ldots,
$$

with $E_{0}(x)=1$. The Euler spline of order $n$ is an extension of $E_{n}$ to all of $\mathbf{R}$ which is obtained via the functional relation

$$
E_{n}(x)=(-1)^{[x]} E_{n}(x-[x]) .
$$

Here, $[x]$ denotes the largest integer contained in $x$. Thus, $E_{n}$ has $n-1$ continuous derivatives on $\mathbf{R}$ and is strictly monotone between its extreme values, which occur at the integers if $n$ is odd and at the half integers if $n$ is even, with

$$
\left\|E_{n}\right\|= \begin{cases}\left|E_{n}(0)\right|=(-1)^{(n+1) / 2} E_{n}(0) & \text { if } n \text { is odd } \\ \left|E_{n}\left(\frac{1}{2}\right)\right|=(-1)^{n / 2} E_{n}\left(\frac{1}{2}\right) & \text { if } n \text { is even. }\end{cases}
$$

$E_{n}(0)$ and $E_{n}\left(\frac{1}{2}\right)$ can be expressed in terms of the Bernoulli and Euler numbers $B_{n+1}$ and $E_{n}$, respectively:

$$
E_{n}(0)=-\frac{2}{n+1}\left(2^{n+1}-1\right) B_{n+1}, \quad E_{n}\left(\frac{1}{2}\right)=\frac{1}{2^{n}} E_{n} .
$$

Values of the Bernoulli and Euler numbers can be found in [14, Table 23.2].

The infinite sum (1.6) is the Fourier expansion of $E_{n}(x)$, so

$$
\varphi_{n}(x)=E_{n}(x) .
$$

The constant $M_{j}$ in (1.5) is equal to the quantity $(j !)^{-1}\left\|E_{n}\right\|$; hence,

$$
M_{j}= \begin{cases}(-1)^{(j-1) / 2} \frac{2}{(j+1) !}\left(2^{j+1}-1\right) B_{j+1} & \text { if } j \text { is odd }, \\ (-1)^{j / 2} \frac{1}{2^{j} j !} E_{j} & \text { if } j \text { is even. }\end{cases}
$$

Because the tables in Kolmogorov's article [5] contain several errors, we give the first few values of $K(n, k)^{n}$, which is rational, and all values of $K(n, k)$ for $n=2, \ldots, 12$ in Tables 1 and 2, respectively. 
TABLE $1 . K(n, k)^{n}$

\begin{tabular}{|c|c|c|c|c|}
\hline \multirow{2}{*}{$n$} & \multicolumn{4}{|c|}{$k$} \\
\cline { 2 - 5 } & 1 & 2 & 3 & 4 \\
\hline 2 & 2 & & & \\
3 & $\frac{9}{8}$ & 3 & & \\
4 & $\frac{512}{375}$ & $\frac{36}{25}$ & $\frac{24}{5}$ & \\
5 & $\frac{1953125}{1572864}$ & $\frac{125}{72}$ & $\frac{225}{128}$ & $\frac{15}{2}$ \\
\hline
\end{tabular}

TABLE 2. $K(n, k)$

\begin{tabular}{|c|c|c|c|c|c|c|c|c|c|c|c|}
\hline \multirow{2}{*}{$n$} & \multicolumn{10}{|c|}{$k$} \\
\cline { 2 - 11 } & 1 & 2 & 3 & 4 & 5 & 6 & 7 & 8 & 9 & 10 & 11 \\
\hline 2 & 1.41421 & & & & & & & & & & \\
3 & 1.04004 & 1.44225 & & & & & & & & & \\
4 & 1.08096 & 1.09545 & 1.48017 & & & & & & & & \\
5 & 1.04426 & 1.11665 & 1.11942 & 1.49628 & & & & & & & \\
6 & 1.04298 & 1.08001 & 1.14520 & 1.13842 & 1.50892 & & & & & & \\
7 & 1.03451 & 1.07289 & 1.10472 & 1.16479 & 1.15137 & 1.51748 & & & & & \\
8 & 1.03086 & 1.06182 & 1.09643 & 1.12407 & 1.18006 & 1.16142 & 1.52408 & & & & \\
9 & 1.02714 & 1.05530 & 1.08336 & 1.11494 & 1.13922 & 1.19197 & 1.16922 & 1.52919 & & & \\
10 & 1.02447 & 1.04945 & 1.07533 & 1.10096 & 1.13001 & 1.15153 & 1.20162 & 1.17551 & 1.53331 & & \\
11 & 1.02220 & 1.04492 & 1.06804 & 1.09198 & 1.11556 & 1.14249 & 1.16169 & 1.20956 & 1.18069 & 1.53668 & \\
12 & 1.02034 & 1.04108 & 1.06227 & 1.08380 & 1.10606 & 1.12788 & 1.15299 & 1.17022 & 1.21622 & 1.18502 & 1.53949 \\
\hline
\end{tabular}

As $n \rightarrow \infty$, we have the asymptotic results

$$
K(n, 1)=1+\frac{1}{n} \ln \frac{4}{\pi}+O\left(\frac{1}{n^{2}}\right)
$$

and

$$
K(n, n-l)=\pi M_{l}\left(1-\frac{l}{n} \ln \frac{4}{\pi}+O\left(\frac{1}{n^{2}}\right)\right), \quad l \text { fixed. }
$$

In particular,

$$
K(n, n-1)=\frac{\pi}{2}\left(1-\frac{1}{n} \ln \frac{4}{\pi}+O\left(\frac{1}{n^{2}}\right)\right) .
$$

Furthermore,

$$
1<K(n, k)<\frac{\pi}{2} .
$$

Given the bounds [14, Equation (23.1.15)] for the Bernoulli and Euler numbers, one may obtain better bounds for the individual $K(n, k)$,

$$
K^{b}(n, k) \leqslant K(n, k) \leqslant K^{\sharp}(n, k),
$$


where

$$
K^{b}(n, k)=\left(\frac{4}{\pi}\right)^{k / n} \frac{\eta_{n-k}}{\varepsilon_{n}^{(n-k) / n}}, \quad K^{\sharp}(n, k)=\left(\frac{4}{\pi}\right)^{k / n} \frac{\varepsilon_{n-k}}{\eta_{n}^{(n-k) / n}},
$$

with

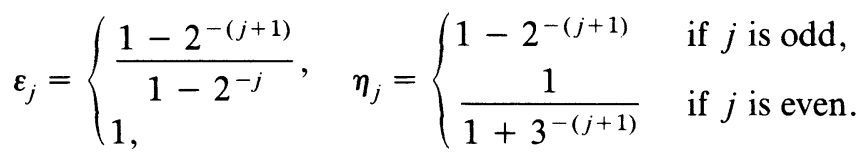

3. General results. The following general results are due to Ditzian [10] and are included here for the sake of completeness.

THEOREM 1. $K(n, k) \leqslant C(n, k)$.

Proof. Let $h$ be fixed, $h>0$. We use the extremal $E_{n}$ for the differential operator $D$ in $L^{\infty}(\mathbf{R})$ to define the sequence $\xi(h) \in l^{\infty}(\mathbf{Z})$,

$$
(\xi(h))_{m}=E_{n}(m h), \quad m \in \mathbf{Z} .
$$

This sequence has the property that $h^{-k}\left\|\Delta^{k} \xi(h)\right\| \rightarrow\left\|E_{n}^{(k)}\right\|$ as $h \rightarrow 0$ for $k=$ $0, \ldots, n$, so the value of the quotient $Q_{n, k}$ for this sequence approaches the value of $Q_{n, k}$ for $E_{n}$, which is $K(n, k)$, as $h$ tends to 0 . Since $C(n, k)$ is the supremum of $Q_{n, k}(\xi)$ over all sequences $\xi \in l^{\infty}(\mathbf{Z})$, we have $Q_{n, k}(\xi(h)) \leqslant C(n, k)$. The assertion of the theorem follows if we let $h$ tend to 0 .

THEOREM 2. $K(n, n-1)=C(n, n-1)$.

Proof. Given an arbitrary sequence $\xi=\left(\xi_{m}\right)_{m \in \mathbf{Z}}$ in $l^{\infty}(\mathbf{Z})$, we define a function $f$ in $L^{\infty}(\mathbf{R})$,

$$
f(x)=\sum_{m} \xi_{m} B_{m, n+1}(x), \quad x \in \mathbf{R},
$$

where $B_{m, n+1}$ is the $B$-spline of order $n+1$ with support $[m, m+n+1]$ (cf. [13, Chapter IX]). This function belongs to $\operatorname{dom} D^{n}$, where $D$ is the differential operator with maximal domain in $L^{\infty}(\mathbf{R})$. Its derivatives are obtained by interpolating the differences of $\xi$,

$$
D^{k} f(x)=\sum_{m}\left(\Delta^{k} \xi\right)_{m-k} B_{m, n+1-k}(x), \quad k=0, \ldots, n .
$$

In particular, $D^{n-1} f$ is the piecewise linear interpolant of $\Delta^{n-1} \xi$,

$$
D^{n-1} f(x)=\sum_{m}\left(\Delta^{n-1} \xi\right)_{m-n+1} B_{m, 2}(x),
$$

and $D^{n} f$ the piecewise constant interpolant of $\Delta^{n} \xi$,

$$
D^{n} f(x)=\sum_{m}\left(\Delta^{n} \xi\right)_{m-n} B_{m, 1}(x) .
$$

Therefore, $\left\|D^{n-1} f\right\|=\left\|\Delta^{n-1} \xi\right\|$ and $\left\|D^{n} f\right\|=\left\|\Delta^{n} \xi\right\|$. Furthermore, $\|f\| \leqslant\|\xi\|$, because the $B$-splines are nonnegative functions that sum to one at every point of $\mathbf{R}$. Thus, using Kolmogorov's result for $D$ with $k=n-1$, we find that

$$
\left\|\Delta^{n-1} \xi\right\| \leqslant K(n, n-1)\|f\|^{1 / n}\left\|D^{n} f\right\|^{(n-1) / n} \leqslant K(n, n-1)\|\xi\|^{1 / n}\left\|\Delta^{n} \xi\right\|^{(n-1) / n} .
$$


Hence, $Q_{n, n-1}(\xi) \leqslant K(n, n-1)$. Taking the supremum over all sequences $\xi \in$ $l^{\infty}(\mathbf{Z})$, we obtain the inequality

$$
C(n, n-1) \leqslant K(n, n-1) .
$$

The reverse inequality follows from Theorem 1.

Equality does not hold for all values of $k$, as is easily shown by a counterexample. Consider the sequence $\xi=\left(\xi_{m}\right)_{m \in \mathbf{Z}}$ with $\xi_{m}=1$ if $m<0, \xi_{m}=-1$ if $m \geqslant 0$. For this sequence we have $Q_{3,1}(\xi)=2^{1 / 3}$, so

$$
K(3,1)=\left(\frac{9}{8}\right)^{1 / 3}<2^{1 / 3} \leqslant C(3,1) .
$$

In fact, we shall show in $\S 6$ that $C(3,1)=2^{1 / 3}$.

4. Lower bounds for $C(n, k)$. In this section we derive lower bounds for $C(n, k)$; we denote them by $C^{b}(n, k)$. In view of Theorem 2 , we can restrict ourselves to pairs $(n, k)$ with $n=3,4, \ldots$ and $k=1, \ldots, n-2$.

Obviously, a lower bound for $C(n, k)$ can be obtained by evaluating the quotient $Q_{n, k}(\xi)$ at any sequence $\xi \in l^{\infty}(\mathbf{Z})$. The following procedure for getting a "good" lower bound was suggested in [11]: Maximize $Q_{n, k}(\xi)$ by choosing $\xi$ from among those sequences in $l^{\infty}(\mathbf{Z})$ for which $\|\xi\|=1$ and $\left\|\Delta^{k} \xi\right\|=2^{k}$. (Clearly, $2^{k}$ is the largest possible value of $\left\|\Delta^{k} \xi\right\|$ if $\|\xi\|=1$.) Sequences of this type must have a core consisting of $k+1$ elements, alternatingly equal to +1 and -1 . One then extends this core in both directions in such a way that $\left\|\Delta^{n} \xi\right\|$ is minimized, while neither $\|\xi\|$ nor $\left\|\Delta^{k} \xi\right\|$ increase beyond the values 1 and $2^{k}$, respectively.

We have implemented this procedure and computed lower bounds $C^{b}(n, k)$ for values of $n$ up to 12 , choosing the elements $\xi_{m}$ from the set $\{-1,0,1\}$. The sequences thus obtained were not unique: In some cases, it was possible to select a sequence with only finitely many nonzero elements; in other cases, an infinite but periodic one resulted. Table 3 contains several examples. In each example, the core is at the center; a type $\mathrm{f}$ indicates a finite sequence, a type $\mathrm{p}$ a periodic one.Values of $C^{b}(n, k)$ thus obtained are given in Table 4 . A superscript * indicates that the given value is greater than the corresponding value of $K(n, k)$.

The algorithm can be worked out analytically for $k=1$ with the result

$$
C^{b}(n, 1)= \begin{cases}2^{(n-1) /(2 n)} & \text { if } n \text { is odd, } \\ 2^{1 / 2} & \text { if } n \text { is even. }\end{cases}
$$

The value of $C^{b}(n, 1)$ thus obtained always exceeds the value of $K(n, 1)$.

TABLE 3. Maximizing sequences $\xi$ for $Q_{n, k} ;\|\xi\|=1$ and $\left\|\Delta^{k} \xi\right\|=2^{k}$.

\begin{tabular}{|c|ccrrrrrrrrrr|c|}
\hline$(n, k)$ & \multicolumn{10}{|c|}{$\xi$} & type \\
\hline$(3,1)$ & $\cdot$ & 0 & 0 & 0 & 1 & 1 & -1 & -1 & 0 & 0 & 0 & $\cdot$ & $\mathrm{f}$ \\
$(4,1)$ & $\cdot$ & 1 & -1 & -1 & 1 & 1 & -1 & -1 & 1 & 1 & -1 &. & $\mathrm{p}$ \\
$(4,2)$ & $\cdot$ & 0 & 0 & 1 & 1 & -1 & 1 & 1 & 0 & 0 & 0 &. & $\mathrm{f}$ \\
$(5,1)$ & $\cdot$ & 1 & -1 & -1 & 1 & 1 & -1 & -1 & 1 & 1 & -1 &. & $\mathrm{p}$ \\
$(5,2)$ & $\cdot$ & 0 & -1 & 1 & 1 & -1 & 1 & 1 & -1 & 0 & 0 &. & $\mathrm{f}$ \\
$(5,3)$ & $\cdot$ & 0 & 0 & 1 & 1 & -1 & 1 & -1 & -1 & 0 & 0 & $\cdot$ & $\mathrm{f}$ \\
\hline
\end{tabular}


TABLE 4. $C^{b}(n, k)$; first pass.

\begin{tabular}{|c|c|c|c|c|c|c|c|c|c|c|}
\hline \multirow[t]{2}{*}{$n$} & \multicolumn{10}{|c|}{$k$} \\
\hline & 1 & 2 & 3 & 4 & 5 & 6 & 7 & 8 & 9 & 10 \\
\hline 3 & $1.25992^{*}$ & & & & & & & & & \\
\hline 4 & $1.41421^{*}$ & $1.15470^{*}$ & & & & & & & & \\
\hline 5 & $1.31951^{*}$ & $1.25878^{*}$ & 1.08342 & & & & & & & \\
\hline 6 & $1.41421^{*}$ & $1.21141^{*}$ & $1.15470^{*}$ & 1.04396 & & & & & & \\
\hline 7 & $1.34590^{*}$ & $1.26642^{*}$ & $1.13122^{*}$ & 1.09046 & 1.02294 & & & & & \\
\hline 8 & $1.41421^{*}$ & $1.22958^{*}$ & $1.18174^{*}$ & 1.07872 & 1.05216 & 1.01188 & & & & \\
\hline 9 & $1.36079^{*}$ & $1.26150^{*}$ & $1.16002^{*}$ & $1.11596^{*}$ & 1.04623 & 1.02971 & 1.00612 & & & \\
\hline 10 & $1.41421^{*}$ & $1.24524^{*}$ & $1.19721^{*}$ & $1.10378^{*}$ & 1.07264 & 1.02670 & 1.01674 & 1.00314 & & \\
\hline 11 & $1.37035^{*}$ & $1.26020^{*}$ & $1.17778^{*}$ & 1.13379* & 1.06583 & 1.04477 & 1.01521 & 1.00934 & 1.00160 & \\
\hline 12 & $1.41421^{*}$ & $1.24460^{*}$ & $1.20605^{*}$ & $1.12199^{*}$ & 1.08874 & 1.04096 & 1.02717 & 1.00855 & 1.00516 & 1.00081 \\
\hline
\end{tabular}

TABLE 5. $Q_{n, k}\left(\xi^{(i)}\right), i=1,2,3$.

\begin{tabular}{|c|c|c|c|c|}
\hline$n$ & $k$ & $Q_{n, k}\left(\xi^{(1)}\right)$ & $Q_{n, k}\left(\xi^{(2)}\right)$ & $Q_{n, k}\left(\xi^{(3)}\right)$ \\
\hline odd & odd & $\left(\frac{4}{3}\right)^{(n-k) /(2 n)}$ & $2^{(n-k) /(2 n)}$ & $<1$ \\
odd & even & $\left(\frac{4}{3}\right)^{(2 n-k) /(2 n)}$ & $<1$ & $2^{k /(2 n)}$ \\
even & odd & $\left(\frac{4}{3}\right)^{(n-2 k) /(2 n)}$ & $2^{1 / 2}$ & $<1$ \\
even & even & $\left(\frac{4}{3}\right)^{(n-k) / n}$ & 1 & 1 \\
\hline
\end{tabular}

Greater lower bounds $C^{b}(n, k)$ may be obtained if the constraint $\left\|\Delta^{k} \xi\right\|=2^{k}$ is relaxed. The periodic sequences

$$
\begin{gathered}
\xi^{(1)}=(\ldots, 1,1,-1,1,1,-1,1,1,-1, \ldots), \\
\xi^{(2)}=(\ldots, 1,1,-1,-1,1,1,-1,-1, \ldots), \\
\xi^{(3)}=(\ldots, 0,1,0,-1,0,1,0,-1, \ldots)
\end{gathered}
$$

still have unit length, but the norms of the vectors of their $k$ th differences are generally less than $2^{k}$,

$$
\begin{gathered}
\left\|\Delta^{k} \xi^{(1)}\right\|=\left(\frac{3}{4}\right)^{[(k-1) / 2]} 2^{k}, \\
\left\|\Delta^{k} \xi^{(2)}\right\|=\left(\frac{1}{2}\right)^{[k / 2]} 2^{k}, \\
\left\|\Delta^{k} \xi^{(3)}\right\|=\left(\frac{1}{2}\right)^{[(k+1) / 2]} 2^{k} .
\end{gathered}
$$

The 3-periodic sequence (4.2) was chosen on the basis of numerical experiments; the 4-periodic sequence (4.3) already appeared in Table 3 for $k=1$; the 4-periodic sequence (4.4) was constructed so that $\Delta \xi^{(3)}=\xi^{(2)}$. The value of $Q_{n, k}$ at these vectors depends on the parities of $n$ and $k$; see Table 5 .

A value of $Q_{n, k}$ less than or equal to one is useless as a lower bound for $C(n, k)$, in view of (2.10) and Theorem 1.

By comparing the entries of Table 5 and selecting the greatest one in each row, we obtain a lower bound for $C(n, k)$ that may, in fact, be greater than the lower bound $C^{b}(n, k)$ given in Table 4 . The result of this exercise is summarized in the following lemma. 
TABLE 6. $C^{b}(n, k)$; second pass.

\begin{tabular}{|c|c|c|c|c|c|c|c|c|c|c|}
\hline \multirow[t]{2}{*}{$n$} & \multicolumn{10}{|c|}{$k$} \\
\hline & 1 & 2 & 3 & 4 & 5 & 6 & 7 & 8 & 9 & 10 \\
\hline 3 & $1.25992^{*}$ & & & & & & & & & \\
\hline 4 & $1.41421^{*}$ & $1.15470^{*}$ & & & & & & & & \\
\hline 5 & $1.31951^{*}$ & $1.25878^{*}$ & $1.14870^{*}$ & & & & & & & \\
\hline 6 & $1.41421^{*}$ & $1.21141^{*}$ & $1.41421^{*}$ & 1.04396 & & & & & & \\
\hline 7 & $1.34590^{*}$ & $1.27965^{*}$ & $1.21901^{*}$ & $1.22812^{*}$ & 1.10409 & & & & & \\
\hline 8 & $1.41421^{*}$ & $1.24081^{*}$ & $1.41421^{*}$ & $1.15470^{*}$ & $1.41421^{*}$ & $1.07457^{*}$ & & & & \\
\hline 9 & $1.36079^{*}$ & $1.29139^{*}$ & $1.25992^{*}$ & $1.25076^{*}$ & $1.16653^{*}$ & $1.25992^{*}$ & 1.08006 & & & \\
\hline 10 & $1.41421^{*}$ & $1.25878^{*}$ & $1.41421^{*}$ & $1.18840^{*}$ & $1.41421^{*}$ & $1.12196^{*}$ & $1.41421^{*}$ & $1.05922^{*}$ & & \\
\hline 11 & $1.37035^{*}$ & $1.29891^{*}$ & $1.28666^{*}$ & $1.26538^{*}$ & $1.20809^{*}$ & $1.23272^{*}$ & 1.13431 & $1.28666^{*}$ & 1.06504 & \\
\hline 12 & $1.41421^{*}$ & $1.27091^{*}$ & $1.41421^{*}$ & $1.21141^{*}$ & $1.41421^{*}$ & $1.15470^{*}$ & $1.41421^{*}$ & 1.10064 & $1.41421^{*}$ & 1.04912 \\
\hline
\end{tabular}

LEMMA 3. $C(n, k) \geqslant C^{b}(n, k)$, where the value of $C^{b}(n, k)$ is obtained from one of the following expressions:

$$
C^{b}(n, k)= \begin{cases}2^{(n-k) /(2 n)} & \text { if } n \text { is odd, } k \text { odd }, \\ \max \left\{2^{k /(2 n)},\left(\frac{4}{3}\right)^{(2 n-k) /(2 n)}\right\} & \text { if } n \text { is odd, } k \text { even, } \\ 2^{1 / 2} & \text { if } n \text { is even, } k \text { odd }, \\ \left(\frac{4}{3}\right)^{(n-k) / n} & \text { if } n \text { is even, } k \text { even, }\end{cases}
$$

or from Table 4, whichever is greater.

We observe that

$$
2^{k /(2 n)} \lessgtr\left(\frac{4}{3}\right)^{(2 n-k) /(2 n)} \quad \text { if } \frac{k}{n} \lessgtr \frac{2 \ln (4 / 3)}{\ln (8 / 3)}=0.58661 .
$$

Table 4 can now be updated; the result is Table 6 . Again, a superscript* indicates that the value of $C^{b}(n, k)$ is greater than that of $K(n, k)$.

Having established these results, we can now investigate when $K(n, k)$ is strictly less than $C(n, k)$.

TheOREM 4. $K(n, k)<C(n, k)$ if $n-k$ is odd, or if $n-k$ is even and either

$$
\frac{k}{n}<\frac{\ln (15 \sqrt{2} / 16)}{\ln (15 \sqrt{2} / 16 \cdot 4 / \pi)}=0.53865 \text { if } n \text { is odd, }
$$

or

$$
\frac{k}{n}<\frac{\ln (81 / 61)}{\ln (81 / 61 \cdot 4 / \pi)}=0.54000 \text { if } n \text { is even. }
$$

PROoF. The proof is based on establishing the strict inequality $K^{\sharp}(n, k)<C^{b}(n, k)$ between the upper bound $K^{\sharp}(n, k)$ of $K(n, k)$, given by (2.12), and the lower bound $C^{b}(n, k)$ of $C(n, k)$, given by (4.8).

(i) $n$ odd, $k$ even. Notice that $n-k$ must be odd, with $n \geqslant 3$ and $n-k \geqslant 3$. Because $\varepsilon_{n-k} \leqslant \varepsilon_{3}=15 / 14$ and $\eta_{n} \geqslant \eta_{3}=15 / 16$, it suffices to establish the inequalities

$$
\left(\frac{4}{\pi}\right)^{k / n} \frac{15}{14}\left(\frac{16}{15}\right)^{(n-k) / n}<(\sqrt{2})^{k / n} \text { if } \frac{k}{n}>\frac{2 \ln (4 / 3)}{\ln (8 / 3)}
$$


and

$$
\left(\frac{4}{\pi}\right)^{k / n} \frac{15}{14}\left(\frac{16}{15}\right)^{(n-k) / n}<\left(\frac{4}{3}\right)^{(2 n-k) /(2 n)} \text { if } \frac{k}{n}<\frac{2 \ln (4 / 3)}{\ln (8 / 3)} .
$$

The first inequality is satisfied as soon as

$$
\frac{k}{n}>\frac{\ln (16 / 14)}{\ln ((16 / 15) \sqrt{2} \cdot(\pi / 4))}
$$

which is always the case if $k / n$ satisfies the inequality in (4.12), and the second inequality is satisfied as soon as

$$
\frac{k}{n}<\frac{\ln (7 / 6)}{\ln (15 /(2 \pi \sqrt{3}))}
$$

which is also always the case if $k / n$ satisfies the inequality in (4.13).

(ii) $n$ even, $k$ odd. Again, $n-k$ must be odd, with $n \geqslant 4$ and $n-k \geqslant 3$. Using the estimates $\varepsilon_{n-k} \leqslant \varepsilon_{3}=15 / 14$ and $\eta_{n} \leqslant \eta_{4}=244 / 243$, we see that it suffices to establish the inequality

$$
\left(\frac{4}{\pi}\right)^{k / n} \frac{15}{14}\left(\frac{243}{244}\right)^{(n-k) / n}<2^{1 / 2}
$$

This inequality is satisfied if

$$
\frac{k}{n}<\frac{\ln ((15 / 14) \sqrt{2} \cdot(244 / 243))}{\ln ((4 / \pi) \cdot(244 / 243))},
$$

which is always the case: The quantity in the right member is greater than one, because $(15 / 14) \sqrt{2}>(4 / \pi)$.

(iii) $n$ odd, $k$ odd. This time, $n-k$ must be even; furthermore, $n \geqslant 3$. Because $\eta_{n} \geqslant \eta_{3}=15 / 16$, it suffices to establish the inequality

$$
\left(\frac{4}{\pi}\right)^{k / n}<\left(\frac{15}{16} \sqrt{2}\right)^{(n-k) / n}
$$

The inequality is satisfied if $k / n$ satisfies (4.10).

(iv) $n$ even, $k$ even. Again, $n-k$ must be even; furthermore, $n \geqslant 4$. Using the estimate $\eta_{n-k} \geqslant \eta_{4}=244 / 243$, we see that it suffices to establish the inequality

$$
\left(\frac{4}{\pi}\right)^{k / n}\left(\frac{244}{243}\right)^{(n-k) / n}<\left(\frac{4}{3}\right)^{(n-k) / n} .
$$

The inequality is satisfied if $k / n$ satisfies (4.11).

The fact that we do not have a good expression for the lower bound of $C(n, k)$ if $n-k$ is even precludes us from extending the theorem to all $(n, k)$.

5. Inequalities for $Q_{n, k}(\xi)$. In this section we derive several inequalities for the quotient $Q_{n, k}(\xi)$. We restrict ourselves again to pairs $(n, k)$ with $n=3,4, \ldots$ and $k=1, \ldots, n-2$. 
LEMMA 5. Let $\xi$ be an arbitrary vector of unit length in $l^{\infty}(\mathbf{Z})$. Then

$$
Q_{n, k}(\xi) \leqslant K(n, k)+\frac{n-k+1}{24} Q_{n, k+2}(\xi)\left\|\Delta^{n} \xi\right\|^{2 / n}
$$

for $k=1, \ldots, n-2$, where $Q_{n, n}(\xi)=1$.

PROOF. Let $f \in L^{\infty}(\mathbf{R})$ be the piecewise polynomial function introduced in (3.2)

$$
f(x)=\sum_{m} \xi_{m} B_{m, n+1}(x), \quad x \in \mathbf{R} .
$$

We recall from the proof of Theorem 2 that $f \in \operatorname{dom} D^{n}$, where $D$ is the differentiation operator with maximal domain in $L^{\infty}(\mathbf{R})$, and

$$
D^{k} f(x)=\sum_{m}\left(\Delta^{k} \xi\right)_{m-k} B_{m, n+1-k}, \quad k=0, \ldots, n .
$$

Furthermore, $\|f\| \leqslant 1$ and $\left\|D^{n} f\right\|=\left\|\Delta^{n} f\right\|$.

For $k=n-l$ and $x \in[m, m+1)$, we have

$$
D^{n-l} f(x)=\sum_{j=m-l}^{m}\left(\Delta^{n-l} \xi\right)_{j-n+l} B_{j, l+1}(x) .
$$

Suppose $l$ is even. If we split off the term with $j=m-n+l / 2$ and use the property that the $B$-splines sum to 1 , we obtain the identity

$$
\begin{aligned}
D^{n-l} f(x)= & \left(\Delta^{n-l} \xi\right)_{m-n+l / 2} \\
& +\sum_{j=m-l}^{m-l / 2-1}\left(\left(\Delta^{n-l} \xi\right)_{j-n+l}-\left(\Delta^{n-l} \xi\right)_{m-n+l / 2}\right) B_{j, l+1}(x) \\
& +\sum_{j=m-l / 2+1}^{m}\left(\left(\Delta^{n-l} \xi\right)_{j-n+l}-\left(\Delta^{n-l} \xi\right)_{m-n+l / 2}\right) B_{j, l+1}(x) .
\end{aligned}
$$

Next, we observe that

$$
B_{m-l+p, l+1}\left(m+\frac{1}{2}\right)=B_{m-p, l+1}\left(m+\frac{1}{2}\right)=B_{0, l+1}\left(p+\frac{1}{2}\right)
$$

for $p=0, \ldots, l / 2-1$. Hence, at $x=m+\frac{1}{2}$, the two sums above can be combined into one:

$$
\begin{aligned}
D^{n-l} f\left(m+\frac{1}{2}\right) & \\
=\left(\Delta^{n-l} \xi\right)_{m-n+l / 2}+\sum_{p=0}^{l / 2-1}\left(\left(\Delta^{n-l} \xi\right)_{m-n+p}-\right. & 2\left(\Delta^{n-l} \xi\right)_{m-n+l / 2} \\
& \left.+\left(\Delta^{n-l} \xi\right)_{m-n+l-p}\right) B_{0, l+1}\left(p+\frac{1}{2}\right) .
\end{aligned}
$$

The linear combination of the $(n-l)$ th differences that occurs under the summation sign can be expressed in terms of $(n-l+2)$ th differences

$$
\begin{aligned}
\left(\Delta^{n-l} \xi\right)_{m-n+p}-2\left(\Delta^{n-l} \xi\right)_{m-n+l / 2}+\left(\Delta^{n-l} \xi\right)_{m-n+l-p} \\
=\sum_{r=1}^{l / 2-1-p} r\left(\left(\Delta^{n-l+2} \xi\right)_{m-n+p+r-1}+\left(\Delta^{n-l+2} \xi\right)_{m-n+l-p-1-r}\right) \\
\quad+\left(\frac{l}{2}-p\right)\left(\Delta^{n-l+2} \xi\right)_{m-n+l / 2-1}
\end{aligned}
$$


Thus

(5.9)

$$
\begin{aligned}
& \left(\Delta^{n-l} \xi\right)_{m-n+l / 2}=D^{n-l} f\left(m+\frac{1}{2}\right) \\
& -\sum_{p=0}^{l / 2-1}\left\{\sum_{r=1}^{l / 2-1-p} r\left(\left(\Delta^{n-l+2} \xi\right)_{m-n+p+r-1}+\left(\Delta^{n-l+2} \xi\right)_{m-n+l-p-1-r}\right)\right. \\
& \left.+\left(\frac{l}{2}-p\right)\left(\Delta^{n-l+2} \xi\right)_{m-n+l / 2-1}\right\} \\
& \quad \times B_{0, l+1}\left(p+\frac{1}{2}\right) .
\end{aligned}
$$

The right-hand side of (5.9) is less in absolute value than the quantity

$$
\begin{aligned}
\left\|D^{n-l} f\right\|+ & \left\{\sum_{p=0}^{l / 2-1}\left(2 \sum_{r=1}^{l / 2-1-p} r+\frac{l}{2}-p\right) B_{0, l+1}\left(p+\frac{1}{2}\right)\right\}\left\|\Delta^{n-l+2} \xi\right\| \\
& =\left\|D^{n-l} f\right\|+\left\{\sum_{p=0}^{l / 2-1}\left(\frac{l}{2}-p\right)^{2} B_{0, l+1}\left(p+\frac{1}{2}\right)\right\}\left\|\Delta^{n-l+2} \xi\right\| .
\end{aligned}
$$

The sum inside the braces can be evaluated

$$
\sum_{p=0}^{l / 2-1}\left(\frac{l}{2}-p\right)^{2} B_{0, l+1}\left(p+\frac{1}{2}\right)=\frac{l+1}{24}
$$

(cf. [15, Theorem 4.21]). Hence,

$$
\left|\left(\Delta^{n-l} \xi\right)_{m-n+l / 2}\right| \leqslant\left\|D^{n-l} f\right\|+\frac{l+1}{24}\left\|\Delta^{n-l+2} \xi\right\| .
$$

As the right-hand side is independent of $m$, we can take the supremum over all $\mathrm{m}$ and arrive at the inequality

$$
\left\|\Delta^{n-l} \xi\right\| \leqslant\left\|D^{n-l} f\right\|+\frac{l+1}{24}\left\|\Delta^{n-l+2} \xi\right\| .
$$

Here, $\left\|D^{n-l} f\right\|$ can be estimated by $K(n, n-l)\|f\|^{l / n}\left\|D^{n} f\right\|^{(n-l) / n}$, according to Kolmogorov, and this bound, in turn, is equal to $K(n, n-l)\left\|\Delta^{n} \xi\right\|^{(n-l) / n}$. Thus,

$$
\left\|\Delta^{n-l} \xi\right\| \leqslant K(n, n-l)\left\|\Delta^{n} \xi\right\|^{(n-l) / n}+\frac{l+1}{24}\left\|\Delta^{n-l+2} \xi\right\| .
$$

The assertion of the lemma now follows upon division of both sides of this inequality by $\left\|\Delta^{n} \xi\right\|^{(n-l) / n}$.

The proof for odd $l$ is similar and is omitted.

Equation (5.1) is a recurrence relation that, upon repeated application, gives an upper bound for $Q_{n, k}(\xi)$ that depends polynomially on $\left\|\Delta^{n} \xi\right\|^{1 / n}$. For example,

$$
\begin{gathered}
Q_{n, n-2}(\xi) \leqslant K(n, n-2)+\frac{1}{8}\left\|\Delta^{n} \xi\right\|^{2 / n} \\
Q_{n, n-3}(\xi) \leqslant K(n, n-3)+\frac{1}{6} K(n, n-1)\left\|\Delta^{n} \xi\right\|^{2 / n}, \\
Q_{n, n-4}(\xi) \leqslant K(n, n-4)+\frac{5}{24}\left(K(n, n-2)+\frac{1}{8}\left\|\Delta^{n} \xi\right\|^{2 / n}\right)\left\|\Delta^{n} \xi\right\|^{2 / n} .
\end{gathered}
$$


These bounds confirm the more qualitative statement of [11, Theorem 5.1]. Obviously, they are most useful when $\left\|\Delta^{n} \xi\right\|$ is small. The following lemma is useful when $\left\|\Delta^{n} \xi\right\|$ is large.

LEMMA 6. Let $\xi$ be an arbitrary vector of unit length in $l^{\infty}(\mathbf{Z})$. Then

$$
Q_{n, k}(\xi) \leqslant \frac{2^{k}}{\left\|\Delta^{n} \xi\right\|^{k / n}}
$$

Proof. The proof is trivial: If $\|\xi\|=1$, then $\left\|\Delta^{k} \xi\right\| \leqslant 2^{k}$.

Lemmas 5 and 6 enable us to estimate $Q_{n, k}(\xi)$ when $\left\|\Delta^{n} \xi\right\|$ is small and large, respectively. Suitable bounds in the intermediate range are derived directly from specific identities among the components of a vector and its $n$th and $k$ th differences. The following lemmas give several examples.

LEMMA 7. Let $\xi$ be an arbitrary vector of unit length in $l^{\infty}(\mathbf{Z})$. Then

$$
\begin{array}{ll}
Q_{n, 1}(\xi) \leqslant \frac{1}{C_{(n-1) / 2}^{n}}\left\{\left\|\Delta^{n} \xi\right\|^{(n-1) / n}+\frac{2^{n}-2 C_{(n-1) / 2}^{n}}{\left\|\Delta^{n} \xi\right\|^{1 / n}}\right\} & \text { if } n \text { is odd, } \\
Q_{n, 1}(\xi) \leqslant \frac{2}{C_{n / 2}^{n+1}}\left\{\left\|\Delta^{n} \xi\right\|^{(n-1) / n}+\frac{2^{n}-C_{n / 2}^{n+1}}{\left\|\Delta^{n} \xi\right\|^{1 / n}}\right\} & \text { if } n \text { is even. }
\end{array}
$$

Here, $C_{p}^{n}$ is the binomial coefficient $\left(\begin{array}{c}n \\ p\end{array}\right)$.

Proof. Suppose $n$ is odd. For any $l \in \mathbf{Z}$, we have the identity

$$
\left(\Delta^{n} \xi\right)_{l}=\sum_{p=0}^{n}(-1)^{p} C_{p}^{n} \xi_{l+n-p} .
$$

The terms with $p=(n-1) / 2$ and $p=(n+1) / 2$ have equal coefficients, but opposite signs. Combining them into one single term involving $(\Delta \xi)_{l+(n-1) / 2}$, we obtain the identity

$$
(\Delta \xi)_{l+(n-1) / 2}=\frac{(-1)^{(n-1) / 2}}{C_{(n-1) / 2}^{n}}\left\{\left(\Delta^{n} \xi\right)_{l}-\sum_{p=0}^{n}{ }^{\prime}(-1)^{p} C_{p}^{n} \xi_{l+n-p}\right\} .
$$

(The prime on the sum indicates that the terms with $p=(n-1) / 2$ and $p=$ $(n+1) / 2$ are to be omitted.) Hence,

$$
\left|(\Delta \xi)_{l+(n-1) / 2}\right| \leqslant \frac{1}{C_{(n-1) / 2}^{n}}\left\{\left\|\Delta^{n} \xi\right\|+\sum_{p=0}^{n} C_{p}^{n}\right\} .
$$

The sum over $p$ is readily evaluated,

$$
\sum_{p=0}^{n}{ }^{\prime} C_{p}^{n}=\sum_{p=0}^{n} C_{p}^{n}-2 C_{(n-1) / 2}^{n}=2^{n}-2 C_{(n-1) / 2}^{n} .
$$

Since the right-hand side of (5.23) is independent of $l$, we can take the supremum over $l$ and find that

$$
\|\Delta \xi\| \leqslant \frac{1}{C_{(n-1) / 2}^{n}}\left\{\left\|\Delta^{n} \xi\right\|+2^{n}-2 C_{(n-1) / 2}^{n}\right\} .
$$

The inequality (5.19) follows upon division of both sides of (5.25) by $\left\|\Delta^{n} \xi\right\|^{1 / n}$. 
The proof for even $n$ is similar. One starts from the identity

$$
\left(\Delta^{n} \xi\right)_{l}-\left(\Delta^{n} \xi\right)_{l+1}=-\sum_{p=0}^{n+1}(-1)^{p} C_{p}^{n+1} \xi_{l+n+1-p}
$$

and proceeds as before, combining the terms with $p=n / 2$ and $p=n / 2+1$. We omit the details.

Lemma 7 is particularly useful to estimate $Q_{n, 1}(\xi)$ in the intermediate range of values of $\left\|\Delta^{n} \xi\right\|$. The quantity in the right member of (5.19) increases indefinitely as $\left\|\Delta^{n} \xi\right\|$ tends to zero and as $\left\|\Delta^{n} \xi\right\|$ tends to infinity, and has a minimum at

$$
\left\|\Delta^{n} \xi\right\|=\frac{1}{n-1}\left(2^{n}-2 C_{(n-1) / 2}^{n}\right) \text {. }
$$

The quantity in the right member of (5.20) behaves similarly, with a minimum at

$$
\left\|\Delta^{n} \xi\right\|=\frac{1}{n-1}\left(2^{n}-C_{n / 2}^{n+1}\right) \text {. }
$$

The inequalities (5.19) and (5.20) are the first of a hierarchy of inequalities that one derives from linear combinations of $n$th differences. For example, if $n$ is odd one may start from the identity

$$
\begin{aligned}
\left(\Delta^{n} \xi\right)_{l-1}+n & \left(\Delta^{n} \xi\right)_{l}+\left(\Delta^{n} \xi\right)_{l+1} \\
& =(n+2) \sum_{p=-1}^{n+1}(-1)^{p} \frac{p(n-p) n !}{(n-p+1) !(p+1) !} \xi_{l+n-p}
\end{aligned}
$$

to derive the inequality

(5.30)

$$
Q_{n, 1}(\xi) \leqslant \frac{1}{C_{(n-3) / 2}^{n}}\left\{\left\|\Delta^{n} \xi\right\|^{(n-1) / n}+\frac{2^{n}-2 C_{(n-3) / 2}^{n}-4\left(2^{n}-1\right) /(n+2)}{\left\|\Delta^{n} \xi\right\|^{1 / n}}\right\},
$$

or, if $n$ is even, from the identity

$$
\begin{aligned}
\left(\Delta^{n} \xi\right)_{l-1}+n\left(\Delta^{n} \xi\right)_{l} & -n\left(\Delta^{n} \xi\right)_{l+1}-\left(\Delta^{n} \xi\right)_{l+2} \\
& =\sum_{p=0}^{n+1}(-1)^{p}(n-p) C_{p}^{n+1}\left(\xi_{l+n-p}-\xi_{l+1+p}\right)
\end{aligned}
$$

to derive the inequality

$$
Q_{n, 1}(\xi) \leqslant \frac{2}{C_{n / 2-1}^{n}}\left\{\left\|\Delta^{n} \xi\right\|^{(n-1) / n}+\frac{2^{n}-C_{n / 2-1}^{n}-2\left(2^{n}-1\right) /(n+1)}{\left\|\Delta^{n} \xi\right\|^{1 / n}}\right\} .
$$

Notice that the linear combination of the elements of $\Delta^{n} \xi$ in the starting identity (5.29) is such that the coefficients of the penultimate elements $\xi_{l+n}$ and $\xi_{l}$ (at $p=0$ and $p=n$, respectively) vanish. A similar remark applies to the linear combination in (5.31). At the next step in the hierarchy, one takes one more element at each end of the vector $\Delta^{n} \xi$ and chooses linear combinations for which the coefficients of the penultimate and a second element of $\xi$ vanish, etc. The same idea is recognized in the construction of the inequalities in Lemmas 8 and 9. 
LEMMA 8. Let $m$ be any nonnegative integer and let $\alpha^{(m)}$ be the vector $\left(\alpha_{l}^{(m)}\right)_{l \in \mathbf{Z}}$ with $\alpha_{l}^{(m)}=0$ if $|l| \geqslant m+1$, and

$$
\alpha_{l}^{(m)}=\left(\begin{array}{c}
m-|l|+3 \\
3
\end{array}\right), \quad l=-m, \ldots, m .
$$

Let $\xi$ be an arbitrary vector of unit length in $l^{\infty}(\mathbf{Z})$. Then

$$
Q_{n, n-2}(\xi) \leqslant \frac{1}{12}(m+1)(m+3)\left\|\Delta^{n} \xi\right\|^{2 / n}+\frac{4}{(m+2)^{2}} \frac{Q_{n, n-4}(\xi)}{\left\|\Delta^{n} \xi\right\|^{2 / n}}
$$

for $n=4,5, \ldots$, where $Q_{n, 0}(\xi)=1$.

Proof. (We suppress the superscript $(m)$ on $\alpha$.) Let $C_{p}^{4}$ be the binomial coefficient $\left(\begin{array}{l}4 \\ p\end{array}\right)$. For any integer $n$ and any $l \in \mathbf{Z}$ we have

$$
\left(\Delta^{n} \xi\right)_{l}=\sum_{p=0}^{4}(-1)^{p} C_{p}^{4}\left(\Delta^{n-4} \xi\right)_{l+p}
$$

Hence,

$$
\begin{aligned}
\sum_{l} \alpha_{l}\left(\Delta^{n} \xi\right)_{l} & =\sum_{l} \sum_{p=0}^{4}(-1)^{p} C_{p}^{4} \alpha_{l}\left(\Delta^{n-4} \xi\right)_{l+p} \\
& =\sum_{l} \sum_{p=0}^{4}(-1)^{p} C_{p}^{4} \alpha_{l-p}\left(\Delta^{n-4} \xi\right)_{l}=\sum_{l}\left(\Delta^{4} \alpha\right)_{l-4}\left(\Delta^{n-4} \xi\right)_{l} .
\end{aligned}
$$

The vector of the fourth differences of $\alpha$ vanishes except for five elements:

$$
\begin{gathered}
\left(\Delta^{4} \alpha\right)_{-m-4}=\left(\Delta^{4} \alpha\right)_{m}=1, \\
\left(\Delta^{4} \alpha\right)_{-3}=\left(\Delta^{4} \alpha\right)_{-1}=-(m+2)^{2}, \\
\left(\Delta^{4} \alpha\right)_{-2}=2(m+2)^{2}-2 .
\end{gathered}
$$

Thus,

$$
\sum_{l} \alpha_{l}\left(\Delta^{n} \xi\right)_{l}=-(m+2)^{2}\left(\Delta^{n-2} \xi\right)_{l}+\left(\Delta^{n-4} \xi\right)_{-m}-2\left(\Delta^{n-4} \xi\right)_{2}+\left(\Delta^{n-4} \xi\right)_{m+4} .
$$

Hence,

$$
\left|\left(\Delta^{n-2} \xi\right)_{1}\right| \leqslant \frac{1}{(m+2)^{2}}\left\{\left(\sum_{l} \alpha_{l}\right)\left\|\Delta^{n} \xi\right\|+4\left\|\Delta^{n-4} \xi\right\|\right\} .
$$

One readily verifies that

$$
\sum_{l} \alpha_{l}=\frac{1}{12}(m+1)(m+2)^{2}(m+3),
$$

so $(5.40)$ reduces to

$$
\left|\left(\Delta^{n-2} \xi\right)_{1}\right| \leqslant \frac{1}{12}(m+1)(m+3)\left\|\Delta^{n} \xi\right\|+\frac{4}{(m+2)^{2}}\left\|\Delta^{n-4} \xi\right\| .
$$


As the reference point for the indices of the elements of $\xi$ is arbitrary, the same estimate holds for any element of $\Delta^{n-2} \xi$, so taking the supremum over all elements we obtain the inequality

$$
\left\|\Delta^{n-2} \xi\right\| \leqslant \frac{1}{12}(m+1)(m+3)\left\|\Delta^{n} \xi\right\|+\frac{4}{(m+2)^{2}}\left\|\Delta^{n-4} \xi\right\| .
$$

The statement of the lemma follows upon division of both sides of this inequality by $\left\|\Delta^{n} \xi\right\|^{(n-2) / n}$.

LEMMA 9. Let $m$ be any nonnegative integer and let $\alpha^{(m)}$ be the vector $\left(\alpha_{l}^{(m)}\right)_{l \in \mathbf{Z}}$ with $\alpha_{l}^{(m)}=0$ if $l \leqslant-(m+1)$ or $l \geqslant m+2$, and

$$
\alpha_{l}^{(m)}=\left\{\begin{array}{cl}
\left(\begin{array}{c}
m+l+4 \\
4
\end{array}\right), & l=-m, \ldots, 0, \\
-\left(\begin{array}{c}
m-l+5 \\
4
\end{array}\right), & l=1, \ldots, m+1
\end{array}\right.
$$

Let $\xi$ be an arbitrary vector of unit lenght in $l^{\infty}(\mathbf{Z})$. Then

$$
\begin{aligned}
Q_{n, n-3}(\xi) \leqslant & \frac{1}{20} \frac{(m+2)(m+4)}{(m+3)}\left\|\Delta^{n} \xi\right\|^{3 / n} \\
& +\frac{(m+2)(m+4)}{(m+1)(m+5)} \frac{Q_{n, n-5}(\xi)}{\left\|\Delta^{n} \xi\right\|^{2 / n}}
\end{aligned}
$$

for $n=4,5, \ldots$, where $Q_{n, 0}(\xi)=1$.

Proof. (We suppress the superscript $(m)$ on $\alpha$.) The proof is similar to the proof of Lemma 8 . Let $C_{p}^{5}$ be the binomial coefficient $\left(\begin{array}{l}5 \\ p\end{array}\right)$. For any integer $n$ and any $l \in \mathbf{Z}$ we have

$$
\left(\Delta^{n} \xi\right)_{l}=\sum_{p=0}^{5}(-1)^{p} C_{p}^{5}\left(\Delta^{n-5} \xi\right)_{l+p}
$$

Hence,

$$
\begin{aligned}
\sum_{l} \alpha_{l}\left(\Delta^{n} \xi\right)_{l} & =\sum_{l} \sum_{p=0}^{5}(-1)^{p} C_{p}^{5} \alpha_{l}\left(\Delta^{n-5} \xi\right)_{l+p} \\
& =\sum_{l} \sum_{p=0}^{5}(-1)^{p} C_{p}^{5} \alpha_{l-p}\left(\Delta^{n-5} \xi\right)_{l}=-\sum_{l}\left(\Delta^{5} \alpha\right)_{l-5}\left(\Delta^{n-5} \xi\right)_{l} .
\end{aligned}
$$

The vector of the fifth differences of $\alpha$ vanishes except for seven elements:

$$
\begin{gathered}
\left(\Delta^{5} \alpha\right)_{-m-5}=\left(\Delta^{5} \alpha\right)_{m+1}=1, \\
\left(\Delta^{5} \alpha\right)_{-4}=\left(\Delta^{5} \alpha\right)_{0}=-\frac{1}{12}(m+2)(m+3)^{2}(m+4), \\
\left(\Delta^{5} \alpha\right)_{-3}=\left(\Delta^{5} \alpha\right)_{-1}=\frac{1}{3}(m+1)(m+3)^{2}(m+5) \\
\left(\Delta^{5} \alpha\right)_{-2}=-\frac{1}{2}(m+1)(m+2)(m+4)(m+5) .
\end{gathered}
$$


Thus,

$$
\begin{aligned}
\sum_{l} \alpha_{l}\left(\Delta^{n} \xi\right)_{l}= & -\frac{1}{3}(m+1)(m+3)^{2}(m+5)\left(\Delta^{n-3} \xi\right)_{2}-\left(\Delta^{n-5} \xi\right)_{-m} \\
& +\frac{1}{12}(m+2)(m+3)^{2}(m+4)\left(\Delta^{n-5} \xi\right)_{1} \\
& -\frac{1}{6}(m+1)\left(m^{2}+6 m+12\right)(m+5)\left(\Delta^{n-5} \xi\right)_{3} \\
& +\frac{1}{12}(m+2)(m+3)^{2}(m+4)\left(\Delta^{n-5} \xi\right)_{5}-\left(\Delta^{n-5} \xi\right)_{m+6}
\end{aligned}
$$

Hence,

$$
\begin{aligned}
\left|\left(\Delta^{n-3} \xi\right)_{2}\right| \leqslant & \frac{1}{\frac{1}{3}(m+1)(m+3)^{2}(m+5)} \\
& \cdot\left\{\left(\sum_{l}\left|\alpha_{l}\right|\right)\left\|\Delta^{n} \xi\right\|+\frac{1}{3}(m+2)(m+3)^{2}(m+4)\left\|\Delta^{n-5} \xi\right\|\right\} .
\end{aligned}
$$

One readily verifies that

$$
\sum_{l}\left|\alpha_{l}\right|=\frac{1}{60}(m+1)(m+2)(m+3)(m+4)(m+5)
$$

so $(5.53)$ reduces to

$$
\left|\left(\Delta^{n-3} \xi\right)_{2}\right| \leqslant \frac{1}{20} \frac{(m+2)(m+4)}{m+3}\left\|\Delta^{n} \xi\right\|+\frac{(m+2)(m+4)}{(m+1)(m+5)}\left\|\Delta^{n-5} \xi\right\| .
$$

As the reference point for the indices of the elements of $\xi$ is arbitrary, the same estimate holds for any element of $\Delta^{n-3} \xi$. Taking the supremum over all elements, we obtain the inequality

$$
\left\|\Delta^{n-3} \xi\right\| \leqslant \frac{1}{20} \frac{(m+2)(m+4)}{(m+3)}\left\|\Delta^{n} \xi\right\|+\frac{(m+2)(m+4)}{(m+1)(m+5)}\left\|\Delta^{n-5} \xi\right\| .
$$

The statement of the lemma follows upon division of both sides of this inequality by $\left\|\Delta^{n} \xi\right\|^{(n-3) / n}$.

In Lemma 8 , the vector $\alpha^{(m)}$ was chosen in such a way that the vector of its fourth differences vanished everywhere except at the extremities (cf. (5.36)) and at the center (cf. (5.37) and (5.38)). The same principle led to the choice of $\alpha^{(m)}$ in Lemma 9. Other choices are, of course, possible. They lead to the same type of inequalities among $Q_{n, n-2}$ and $Q_{n, n-4}$ (in the case of Lemma 8) or $Q_{n, n-3}$ and $Q_{n, n-5}$ (in the case of Lemma 9), but with different coefficients. These new inequalities may be better over some ranges of $\left\|\Delta^{n} \xi\right\|$, as we shall see in the following section.

6. Values of $C(n, k)$. In this section we compute $C(n, k), k=0, \ldots, n-1$, for $n=2, \ldots, 5$. The results are summarized in Table 7. The entries for $k=n-1$ are the same as in Table 2, in accordance with Theorem 2.

6.1. $C(3,1)=2^{1 / 3}=1.25992$. From Lemma 3 we know that $C(n, k) \geqslant 2^{1 / 3}$, so it suffices to establish the reverse inequality.

Let $\xi$ be an arbitrary vector of unit length in $l^{\infty}(\mathbf{Z})$. From $(5.15)$ we have the estimate

$$
Q_{3,1}(\xi) \leqslant\left(\frac{9}{8}\right)^{1 / 3}+\frac{1}{8}\left\|\Delta^{3} \xi\right\|^{2 / 3}
$$


from (5.18) the estimate

$$
Q_{3,1}(\xi) \leqslant 2 /\left\|\Delta^{3} \xi\right\|^{1 / 3},
$$

and from (5.19) the estimate

$$
Q_{3,1}(\xi) \leqslant \frac{1}{3}\left\|\Delta^{3} \xi\right\|^{2 / 3}+\frac{2}{3} /\left\|\Delta^{3} \xi\right\|^{1 / 3} .
$$

The inequality $Q_{3,1}(\xi) \leqslant 2^{1 / 3}$ follows

(i) from (6.1) if $0 \leqslant\left\|\Delta^{3} \xi\right\| \leqslant 8\left(4^{2 / 3}-3^{2 / 3}\right)^{3 / 2}=2.33298$,

(ii) from (6.3) if $3 \sqrt{2}-5 / 2=1.74264 \leqslant\left\|\Delta^{3} \xi\right\| \leqslant 4$, and

(iii) from (6.2) if $\left\|\Delta^{3} \xi\right\| \geqslant 4$.

Hence, $Q_{3,1}(\xi)$ is uniformly bounded by $2^{1 / 3}$ over the entire range of $\left\|\Delta^{3} \xi\right\|$. It follows that $C(3,1) \leqslant 2^{1 / 3}$.

TABLE 7. $C(n, k)$. The entries for $C(5,3)$ represent upper and lower bounds.

\begin{tabular}{|c|c|c|c|c|}
\hline \multirow{2}{*}{$n$} & \multicolumn{4}{|c|}{$k$} \\
\cline { 2 - 5 } & 1 & 2 & 3 & 4 \\
\hline 2 & 1.41421 & & & \\
3 & 1.25992 & 1.44225 & & \\
4 & 1.41421 & 1.15470 & 1.48017 & \\
5 & 1.31951 & 1.25878 & 1.19758 & 1.49628 \\
\hline
\end{tabular}

6.2. $C(4,1)=2^{1 / 2}=1.41421$. From Lemma 3 we know that $C(4,1) \geqslant 2^{1 / 2}$, so it suffices to establish the reverse inequality.

Let $\xi$ be an arbitrary vector of unit length in $l^{\infty}(\mathbf{Z})$. From (5.16) we have the estimate

$$
Q_{4,1}(\xi) \leqslant\left(\frac{512}{375}\right)^{1 / 4}+\frac{1}{6}\left(\frac{24}{5}\right)^{1 / 4}\left\|\Delta^{4} \xi\right\|^{2 / 4},
$$

from (5.18) the estimate

$$
Q_{4,1}(\xi) \leqslant 2 /\left\|\Delta^{4} \xi\right\|^{1 / 4}
$$

and from (5.20) the estimate

$$
Q_{4,1}(\xi) \leqslant \frac{1}{5}\left\|\Delta^{4} \xi\right\|^{3 / 4}+\frac{6}{5} /\left\|\Delta^{4} u \xi\right\|^{1 / 4} .
$$

The inequality $Q_{4,1}(\xi) \leqslant 2^{1 / 2}$ follows

(i) from $(6.4)$ if $0 \leqslant\left\|\Delta^{4} \xi\right\| \leqslant\left(\frac{72}{25}\right)^{1 / 2}\left(375^{1 / 4}-128^{1 / 4}\right)^{2}=1.82487$,

(ii) from (6.6) if $0.91414 \leqslant\left\|\Delta^{4} \xi\right\| \leqslant 4$, and

(iii) from (6.5) if $\left\|\Delta^{4} \xi\right\| \geqslant 4$.

Hence, $Q_{4,1}(\xi)$ is uniformly bounded by $2^{1 / 2}$ over the entire range of $\left\|\Delta^{4} \xi\right\|$. It follows that $C(4,1) \leqslant 2^{1 / 2}$.

6.3. $C(4,2)=\left(\frac{4}{3}\right)^{1 / 2}=1.15470$. From Lemma 3 we know that $C(4,2) \geqslant\left(\frac{4}{3}\right)^{1 / 2}$, so it suffices to establish the reverse inequality.

Let $\xi$ be an arbitrary vector of unit length in $l^{\infty}(\mathbf{Z})$. From (5.15) we have the estimate

$$
Q_{4,2}(\xi) \leqslant\left(\frac{36}{25}\right)^{1 / 4}+\frac{1}{8}\left\|\Delta^{4} \xi\right\|^{2 / 4},
$$


from (5.18) the estimate

$$
Q_{4,2}(\xi) \leqslant 4 /\left\|\Delta^{4} \xi\right\|^{2 / 4},
$$

and from (5.33), taking $m=0,1,2$, the estimates

$$
\begin{aligned}
& Q_{4,2}(\xi) \leqslant \frac{1}{4}\left\|\Delta^{4} \xi\right\|^{2 / 4}+1 /\left\|\Delta^{4} \xi\right\|^{2 / 4}, \\
& Q_{4,2}(\xi) \leqslant \frac{2}{3}\left\|\Delta^{4} \xi\right\|^{2 / 4}+\frac{4}{9} /\left\|\Delta^{4} \xi\right\|^{2 / 4},
\end{aligned}
$$

and

$$
Q_{4,2}(\xi) \leqslant \frac{5}{4}\left\|\Delta^{4} \xi\right\|^{2 / 4}+\frac{1}{4} /\left\|\Delta^{4} \xi\right\|^{2 / 4},
$$

respectively. The inequality $Q_{4,2}(\xi) \leqslant\left(\frac{4}{3}\right)^{1 / 2}$ follows

(i) from (6.7) if $0 \leqslant\left\|\Delta^{4} \xi\right\| \leqslant \frac{128}{15}(\sqrt{10}-3)^{2}=0.22472$,

(ii) from (6.11) if $\frac{3}{25}=0.12 \leqslant\left\|\Delta^{4} \xi\right\| \leqslant \frac{1}{3}$,

(iii) from (6.10) if $\frac{1}{3} \leqslant\left\|\Delta^{4} \xi\right\| \leqslant \frac{4}{3}$,

(iv) from (6.9) if $\frac{4}{3} \leqslant\left\|\Delta^{4} \xi\right\| \leqslant 12$, and

(v) from (6.8) if $\left\|\Delta^{4} \xi\right\| \geqslant 12$.

Hence, $Q_{4,2}(\xi)$ is uniformly bounded by $\left(\frac{4}{3}\right)^{1 / 2}$ over the entire range of $\left\|\Delta^{4} \xi\right\|$. It follows that $C(4,2) \leqslant\left(\frac{4}{3}\right)^{1 / 2}$.

We observe that, although Ditzian and Newman give the correct value of $C(4,2)$ in [11], their proof is incomplete.

6.4. $C(5,1)=2^{2 / 5}=1.31951$. From Lemma 3 we know that $C(5,1) \geqslant 2^{2 / 5}$, so it suffices to establish the reverse inequality.

Let $\xi$ be an arbitrary vector of unit length in $l^{\infty}(\mathbf{Z})$. From (5.17) we have the estimate

$$
Q_{5,1}(\xi) \leqslant\left(\frac{1953125}{1572864}\right)^{1 / 5}+\frac{5}{24}\left(\frac{225}{128}\right)^{1 / 5}\left\|\Delta^{5} \xi\right\|^{2 / 5}+\frac{5}{192}\left\|\Delta^{5} \xi\right\|^{4 / 5}
$$

from (5.18) the estimate

$$
Q_{5,1}(\xi) \leqslant 2 /\left\|\Delta^{5} \xi\right\|^{1 / 5}
$$

and from (5.19) the estimate

$$
Q_{5,1}(\xi) \leqslant \frac{1}{10}\left\|\Delta^{5} \xi\right\|^{4 / 5}+\frac{6}{5} /\left\|\Delta^{5} \xi\right\|^{1 / 5} .
$$

The inequality $Q_{5,1}(\xi) \leqslant 2^{2 / 5}$ follows

(i) from (6.12) if $0 \leqslant\left\|\Delta^{5} \xi\right\| \leqslant 1.14534$,

(ii) from (6.14) if $0.97684 \leqslant\left\|\Delta^{5} \xi\right\| \leqslant 8$, and

(iii) from (6.13) if $\left\|\Delta^{5} \xi\right\| \geqslant 8$.

Hence, $Q_{5,1}(\xi)$ is uniformly bounded by $2^{2 / 5}$ over the entire range of $\left\|\Delta^{5} \xi\right\|$. It follows that $C(5,1) \leqslant 2^{2 / 5}$.

6.5. $C(5,2)=\left(\frac{4}{3}\right)^{4 / 5}=1.25878$. From Lemma 3 we know that $C(5,2) \geqslant\left(\frac{4}{3}\right)^{4 / 5}$, so it suffices to establish the reverse inequality.

Let $\xi$ be an arbitrary vector of unit length in $l^{\infty}(\mathbf{Z})$. From (5.16) we have the estimate

$$
Q_{5,2}(\xi) \leqslant\left(\frac{125}{72}\right)^{1 / 5}+\frac{1}{6}\left(\frac{15}{2}\right)^{1 / 5}\left\|\Delta^{5} \xi\right\|^{2 / 5}
$$


from (5.18) the estimate

$$
Q_{5,2}(\xi) \leqslant 4 /\left\|\Delta^{5} \xi\right\|^{2 / 5},
$$

and from (5.45), taking $m=0$ and $m=1$, the estimates

$$
Q_{5,2}(\xi) \leqslant \frac{2}{15}\left\|\Delta^{5} \xi\right\|^{3 / 5}+\frac{8}{5} /\left\|\Delta^{5} \xi\right\|^{2 / 5},
$$

and

$$
Q_{5,2}(\xi) \leqslant \frac{3}{16}\left\|\Delta^{5} \xi\right\|^{3 / 5}+\frac{5}{4} /\left\|\Delta^{5} \xi\right\|^{2 / 5},
$$

respectively. Furthermore,

$$
\begin{gathered}
Q_{5,2}(\xi) \leqslant \frac{38}{75}\left\|\Delta^{5} \xi\right\|^{3 / 5}+\frac{2}{3} /\left\|\Delta^{5} \xi\right\|^{2 / 5}, \\
Q_{5,2}(\xi) \leqslant \frac{154}{243}\left\|\Delta^{5} \xi\right\|^{3 / 5}+\frac{140}{243} /\left\|\Delta^{5} \xi\right\|^{2 / 5},
\end{gathered}
$$

and

$$
Q_{5,2}(\xi) \leqslant \frac{96}{77}\left\|\Delta^{5} \xi\right\|^{3 / 5}+\frac{4}{11} /\left\|\Delta^{5} \xi\right\|^{2 / 5} .
$$

(The inequalities (6.19) through (6.21) are obtained as in Lemma 9, with

$$
\begin{aligned}
& \alpha^{(2)}=\left(\begin{array}{lll}
\cdots & 31520-20-15-30 \cdots),
\end{array}\right.
\end{aligned}
$$

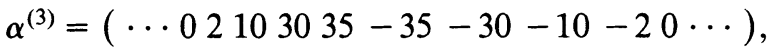

and

$$
\alpha^{(4)}=(\cdots 052575126105-105-126-75-25-50 \cdots),
$$

respectively; cf. the remark following Lemma 9. We omit the details.)

The inequality $Q_{5,2}(\xi) \leqslant\left(\frac{4}{3}\right)^{4 / 5}$ follows

(i) from (6.15) if $0 \leqslant\left\|\Delta^{5} \xi\right\| \leqslant 0.24526$,

(ii) from (6.21) if $0.08502 \leqslant\left\|\Delta^{5} \xi\right\| \leqslant 0.42606$,

(iii) from (6.20) if $0.27356 \leqslant\left\|\Delta^{5} \xi\right\| \leqslant 1.29047$,

(iv) from (6.19) if $0.39143 \leqslant\left\|\Delta^{5} \xi\right\| \leqslant 1.88721$,

(v) from (6.18) if $1.77068 \leqslant\left\|\Delta^{5} \xi\right\| \leqslant 10.58443$,

(vi) from (6.17) if $3.39637 \leqslant\left\|\Delta^{5} \xi\right\| \leqslant 18$, and

(vii) from (6.16) if $\left\|\Delta^{5} \xi\right\| \geqslant 18$.

Hence, $Q_{5,2}(\xi)$ is uniformly bounded by $\left(\frac{4}{3}\right)^{4 / 5}$ over the entire range of $\left\|\Delta^{5} \xi\right\|$. It follows that $C(5,2) \leqslant\left(\frac{4}{3}\right)^{4 / 5}$.

6.6. $2^{1 / 5}=1.14870 \leqslant C(5,3) \leqslant 1.19758$. We have been unsuccessful in finding the value of the best possible constant $C(n, k)$ in the case $n=5, k=3$. It is not clear whether this is due to the lack of a good lower bound for $C(n, k)$ or to the difficulty in obtaining an upper bound for $Q_{n, k}(\xi)$ in the intermediate range of $\left\|\Delta^{5} \xi\right\|$-values.

From Lemma 3 we know that $C(5,3) \geqslant 2^{1 / 5}$. The upper bound given above is obtained in the following way. 
Let $\xi$ be an arbitrary vector of unit length in $l^{\infty}(\mathbf{Z})$. From (5.15) we have the estimate

$$
Q_{5,3}(\xi) \leqslant\left(\frac{225}{128}\right)^{1 / 5}+\frac{1}{8}\left\|\Delta^{5} \xi\right\|^{2 / 5}
$$

and from (5.18) the estimate

$$
Q_{5,3}(\xi) \leqslant 8 /\left\|\Delta^{5} \xi\right\|^{3 / 5} \text {. }
$$

We observe that the constant term in the right member of (6.22) is only slightly less than $2^{1 / 5}$, so the interval over which (6.22) can be used to estimate $Q_{5,3}(\xi)$ by $2^{1 / 5}$ is very small; in fact, it extends only up to the point

$$
\left\|\Delta^{5} \xi\right\|=2^{4}\left(256^{1 / 5}-225^{1 / 5}\right)^{5 / 2}=0.026543 .
$$

On the other hand, (6.23) yields $Q_{5,3}(\xi) \leqslant 2^{1 / 5}$ only for $\left\|\Delta^{5} \xi\right\| \geqslant 2^{14 / 3}=25.39842$. $Q_{5,3}(\xi)$ therefore remains to be estimated over a rather wide gap, for which a variety of inequalities is needed.

The inequality (5.33) requires good estimates for $Q_{5,1}(\xi)$. We found the following ones useful, besides (6.14):

$$
\begin{aligned}
& Q_{5,1}(\xi) \leqslant \frac{1}{5}\left\|\Delta^{5} \xi\right\|^{4 / 5}+\frac{6}{7} /\left\|\Delta^{5} \xi\right\|^{1 / 5}, \\
& Q_{5,1}(\xi) \leqslant \frac{1}{3}\left\|\Delta^{5} \xi\right\|^{4 / 5}+\frac{62}{81} /\left\|\Delta^{5} \xi\right\|^{1 / 5} .
\end{aligned}
$$

The former is obtained from (5.30); the latter is derived by the method of Lemma 8 , with

$$
\alpha^{(2)}=(\cdots 01515510 \cdots) \text {. }
$$

Thus we obtain from (5.33), taking $m=0$ and using (6.14) and (6.24), the inequalities

$$
Q_{5,3}(\xi) \leqslant \frac{7}{20}\left\|\Delta^{5} \xi\right\|^{2 / 5}+\frac{6}{5} /\left\|\Delta^{5} \xi\right\|^{3 / 5}
$$

and

$$
Q_{5,3}(\xi) \leqslant \frac{9}{20}\left\|\Delta^{5} \xi\right\|^{2 / 5}+\frac{6}{7} /\left\|\Delta^{5} \xi\right\|^{3 / 5},
$$

respectively; also from (5.33), taking $m=1$ and using (6.24) and (6.25), the inequalities

$$
Q_{5,3}(\xi) \leqslant \frac{34}{45}\left\|\Delta^{5} \xi\right\|^{2 / 5}+\frac{8}{21} /\left\|\Delta^{5} \xi\right\|^{3 / 5}
$$

and

$$
Q_{5,3}(\xi) \leqslant \frac{22}{27}\left\|\Delta^{5} \xi\right\|^{2 / 5}+\frac{248}{729} /\left\|\Delta^{5} \xi\right\|^{3 / 5},
$$

respectively. Furthermore,

$$
Q_{5,3}(\xi) \leqslant \frac{1}{5}\left\|\Delta^{5} \xi\right\|^{2 / 5}+\frac{12}{5} /\left\|\Delta^{5} \xi\right\|^{3 / 5},
$$

and

$$
Q_{5,3}(\xi) \leqslant \frac{3}{5}\left\|\Delta^{5} \xi\right\|^{2 / 5}+\frac{4}{7} /\left\|\Delta^{5} \xi\right\|^{3 / 5} \text {. }
$$


(The inequalities (6.30) and (6.31) are obtained as in Lemma 8, with

$$
\alpha^{(0)}=(\cdots 010 \cdots)
$$

and

$$
\alpha^{(1)}=(\cdots 01510 \cdots),
$$

respectively. We omit the details.)

The least upper bound for $Q_{5,3}(\xi)$ is obtained

(i) from (6.22) if $0 \leqslant\left\|\Delta^{5} \xi\right\| \leqslant 0.30909$, where its maximum value 1.19758 is achieved at the right endpoint,

(ii) from (6.29) if $0.30909 \leqslant\left\|\Delta^{5} \xi\right\| \leqslant 0.68783$, where its maximum value 1.19758 is achieved at the left endpoint,

(iii) from (6.28) if $0.68783 \leqslant\left\|\Delta^{5} \xi\right\| \leqslant 1.22449$, where its maximum value 1.15667 is achieved at the right endpoint,

(iv) from (6.31) if $1.22449 \leqslant\left\|\Delta^{5} \xi\right\| \leqslant 1.90476$, where its maximum value 1.16461 is achieved at the right endpoint, and

(v) from (6.27) if $1.90476 \leqslant\left\|\Delta^{5} \xi\right\| \leqslant 2.27212$, where its maximum value 1.16461 is achieved at the left endpoint.

For values of $\left\|\Delta^{5} \xi\right\|$ beyond 2.27212 we use the rough estimate $Q_{5,3}(\xi) \leqslant 2^{1 / 5}=$ 1.14870 , which follows

(vi) from (6.27) if $2.27212 \leqslant\left\|\Delta^{5} \xi\right\| \leqslant 3.60558$,

(vii) from (6.26) if $3.34806 \leqslant\left\|\Delta^{5} \xi\right\| \leqslant 8$,

(viii) from (6.30) if $8 \leqslant\left\|\Delta^{5} \xi\right\| \leqslant 42.39816$, and

(ix) from (6.23) if $\left\|\Delta^{5} \xi\right\| \geqslant 25.39842$.

All other inequalities which we have investigated yield upper bounds for $Q_{5,3}(\xi)$ that are greater than or equal to the ones given above. We therefore conclude that the least upper bound of $Q_{5,3}(\xi)$ over the entire range of $\left\|\Delta^{5} \xi\right\|$ is 1.19758 . As we have not been able to determine whether there exists a sequence $\xi$ in $l^{\infty}(\mathbf{Z})$ for which $Q_{5,3}(\xi)=1.19758$, our analysis proves only the inequality $C(5,3) \leqslant 1.19758$.

ACKNowledgment. We thank Professor A. Zettl (Northern Illinois University) for suggesting the study of norm inequalities for the difference operator, Professor Man Kam Kwong (Northern Illinois University) for providing us with several useful inequalities, and Dr. M. Minkoff (Argonne National Laboratory) for contributing toward our understanding of spline functions.

\section{REFERENCES}

1. J. Hadamard, Sur le module maximum d'une fonction et de ses dérivées, C. R. Acad. Sci. Paris Ser. A 42 (1914), 68-72.

2. E. Landau, Einige Ungleichungen für zweimal differentiierbare Funktionen, Proc. London Math. Soc. 13 (1913), 43-49.

3. G. E. Šilov, On inequalities between derivatives, Moskov. Gos. Univ. Sb. Rabot Stud. Nauchn. Kruzhkov (1937), 17-27. (Russian)

4. A. N. Kolmogorov, Dokl. Akad. Nauk SSSR (N.S.) 15 (1937), 107-112.

5. on an infinite interval, Moscov. Gos. Univ. Uchen. Zap. Mat. 30 (1939), 3-13; English transl., Amer. Math. Soc. Transl. 4 (1949); reprint, Amer. Math. Soc. Transl. (1) 2 (1962), 233-243.

6. Ju. I. Ljubič, On the belonging of the powers of an operator of a given vector to a certain linear class, Dokl. Akad. Nauk SSSR 102 (1955), 881-884. (Russian) 
7. (1960), 825-864; English transl., Amer. Math. Soc. Transl. (2) 40 (1964), 39-84.

8. H. A. Gindler and J. A. Goldstein, Dissipative operator versions of some classical inequalities, J. Analyse Math. 28 (1975), 213-238.

9. __ Dissipative operators and series inequalities, Bull. Austral. Math. Soc. 23 (1981), 429-442.

10. Z. Ditzian, Discrete and shift Kolmogorov type inequalities, Proc. Roy. Soc. Edinburgh A 93 (1983), 307-313.

11. Z. Ditzian and D. J. Newman, Discrete Kolmogorov-type inequalities, Preprint (1984).

12. I. J. Schoenberg, Cardinal spline interpolation, CBMS 12, SIAM, Philadelphia, Pa., 1973.

13. C. de Boor, A practical guide to splines, Applied Mathematical Sciences, no. 27, Springer-Verlag, New York, 1978.

14. M. Abramowitz and I. A. Stegun (eds.), Handbook of mathematical functions, NBS Applied Mathematics Series, no. 55, Washington, D. C., 1964.

15. L. L. Schumaker, Spline functions: Basic theory. Wiley, New York, 1981.

Mathematics and Computer Science Division, Argonne National laboratory, Argonne, ILLINOIS 60439 